\title{
Systematic review of anti-inflammatory agents for the management of oral mucositis in cancer patients
}

\author{
Ourania Nicolatou-Galitis • Triantafyllia Sarri • Joanne Bowen • Mario Di Palma • \\ Vassilios E. Kouloulias • Pasquale Niscola • Dorothea Riesenbeck • Monique Stokman • \\ Wim Tissing • Eric Yeoh • Sharon Elad • Rajesh V. Lalla $\cdot$ For The Mucositis Study \\ Group of the Multinational Association of Supportive Care in Cancer/International Society of Oral Oncology \\ (MASCC/ISOO)
}

Received: 3 April 2013 / Accepted: 7 May 2013 / Published online: 24 May 2013

(C) Springer-Verlag Berlin Heidelberg 2013

\begin{abstract}
Purpose The aim of this project was to review the available literature and define clinical practice guidelines for the use of anti-inflammatory agents for the prevention and treatment of oral mucositis in cancer patients.

Materials and methods A systematic review was conducted by the Mucositis Study Group of the Multinational Association of Supportive Care in Cancer/International Society of Oral Oncology. The body of evidence for use of each intervention, in each cancer treatment setting, was assigned an evidence level. Based on the evidence level, one of the following three guideline determinations was possible: recommendation, suggestion, and no guideline possible.
\end{abstract}

\footnotetext{
O. Nicolatou-Galitis $(\bowtie) \cdot T$. Sarri

Oral Oncology Unit, Clinic of Hospital Dentistry, Dental School, University of Athens, Bouboulinas 41, N. Psyhico,

154 51, Athens, Greece

e-mail: nicolatou.galitis@lycos.com

J. Bowen • E. Yeoh

School of Medical Sciences, University of Adelaide, Adelaide,

South Australia, Australia

M. Di Palma

Ambulatory Department, Institut Gustave Roussy, Villejuif, France

V. E. Kouloulias

2nd Department of Radiology, Medical School, Attikon Hospital,

Athens, Greece

P. Niscola

Hematology Unit, St. Eugenio Hospital, Rome, Italy

D. Riesenbeck

Radiation Oncology Practice, Recklinghausen, Germany
}

Results Forty-one papers were reviewed. There was sufficient evidence to recommend the use of benzydamine mouthwash for the prevention of oral mucositis in head and neck cancer patients receiving moderate-dose radiation therapy (up to $50 \mathrm{~Gy}$ ), without concomitant chemotherapy. A new suggestion was developed against the use of misoprostol mouthwash for the prevention of oral mucositis in head and neck cancer patients receiving radiation therapy. Positive results were reported for some other anti-inflammatory agents. However, no guidelines were able to be developed for any other agents due to insufficient and/or conflicting evidence.

Conclusions The use of anti-inflammatory agents continues to be a promising strategy for the prevention and treatment of oral mucositis. Additional well-designed studies are

\footnotetext{
M. Stokman

Department of Oral and Maxillofacial Surgery, University Medical Center Groningen, University of Groningen, Groningen, The Netherlands

W. Tissing

Department of Pediatric Oncology, University Medical Center Groningen, University of Groningen, Groningen, The Netherlands

E. Yeoh

Radiation Oncology Department, Royal Adelaide Hospital, Adelaide, Australia

S. Elad

Division of Oral Medicine, Eastman Institute for Oral Health, University of Rochester Medical Center, Rochester, NY, USA

R. V. Lalla

Section of Oral Medicine and Neag Comprehensive Cancer Center, University of Connecticut Health Center, Farmington, CT, USA
} 
needed to examine the use of this class of agents for oral mucositis.

Keywords Anti- inflammatory agents · Guidelines · Systematic review $\cdot$ Oral mucositis

\section{Introduction}

Considerable evidence has indicated that the inflammatory response to radiation and/or chemotherapy plays an important role in the pathogenesis of oral mucositis [1-3]. Locally produced cytokines can amplify tissue injury. Bacterial colonization of oral mucosal ulcerations can result in the flow of endotoxin (lipopolysaccharides) into the submucosal tissue causing the release of additional cytokines and the increase of tissue toxicity. This understanding of the pathobiology of oral mucositis has led to the evaluation of several agents targeting key components of the radiation- and chemotherapy-induced inflammatory response.

In May 2004, the Mucositis Study Group of the Multinational Association of Supportive Care in Cancer/ International Society of Oral Oncology (MASCC/ISOO) first published evidence-based clinical practice guidelines on the prevention and treatment of oral mucositis [4]. This review examined the literature from January 1966 to May 2002. Due to the exponential increase in mucositis-related research, a need for updating this literature review and guidelines process was critical, so an update was conducted in June of 2005 [5, 6]. Sufficient clinical evidence supported a recommendation for the use of benzydamine mouthwash for the prevention of oral mucositis in patients with head and neck cancer receiving moderate-dose radiation therapy [4-6]. Small numbers of study subjects and varying quality of the studies did not provide enough evidence to support any additional guidelines regarding the use of any other anti-inflammatory agents in the management of oral mucositis [5].

As part of a comprehensive update of the MASCC/ISOO clinical practice guidelines for mucositis, the aim of this project was to systematically review the available literature from January 1966 to December 31st 2010, and define updated clinical practice guidelines for the use of antiinflammatory agents for the prevention and treatment of oral mucositis in cancer patients.

\section{Methods}

The methods are described in detail in Bowen et al. [7] and Elad et al. [8]. Briefly, a literature search was conducted for relevant papers indexed in Medline from 1966 to December 31st, 2010. The intervention keywords used for the literature search were as follows: Aminosalicylic acid, Amifostine, Amlexanox, Anti-inflammatory, Anti-TNF, Anti-tumor necrosis factor, Aspirin, Benadryl, Benzydamine, Betamethasone, Celecoxib, Corticosteroid, Dexamethasone, Diphenhydramine, Ethyol, Flurbiprofen, Histamine, Hydrocortisone, Ibuprofen, Indomethacin, Infliximab, Irsogladine, Lactoferrin, Mesalazine, Misoprostol, N-Acetyl cysteine, Non-steroidal anti-inflammatory agents, NSAIDS, Orgotein, Prednisone, Prostaglandin, RK-02-02, Salicylic acid, Steroid, Thalidomide, TNF antibody, TNF inhibitor, and Tumor necrosis factor/TNF. Articles that evaluated anti-inflammatory agents for the prevention and/or treatment of cancer therapy-induced oral mucositis in humans were selected for review. The detailed inclusion and exclusion criteria are presented in Bowen et al. [7]. Each paper was reviewed by two independent reviewers. The large number of papers was divided among 12 calibrated reviewers to keep the workload reasonable. Data was extracted using a standard electronic form. Studies were evaluated based on the list of major and minor flaws published by Hadorn [9]. A Level of Evidence was assigned for each intervention based on the Somerfield criteria [10]. A welldesigned study was defined as a study with no major flaws per the Hadorn criteria. Findings from the reviewed studies were integrated into guidelines based on the overall Level of Evidence for each intervention. Guidelines were classified into three types: recommendation, suggestion, and no guideline possible. Guidelines were separated based on (1) the aim of the intervention (prevention or treatment of oral mucositis), (2) the treatment modality [head and neck radiotherapy, head and neck chemoradiotherapy, standard dose chemotherapy, and high-dose chemotherapy prior to hematopoetic stem cell transplant (HSCT)], and (3) the route of administration of the intervention (intravenous, intramuscular, systemic per os, and topical application).

\section{Results}

The literature searches identified 908 articles. Of those, 759 articles were excluded after evaluating the title and abstract. Articles that did not report the effects of an intervention on oral mucositis or on related outcomes, animal or in vitro studies, and papers published in a language other than English were excluded. Forty-three review papers were further excluded after detailed evaluation of their title and abstract. The remaining 106 articles were assessed in detail. Of these 40 were related to amifostine and 66 to other antiinflammatory agents. Due to the large number of articles related to amifostine, the results related to that agent have been published in a separate manuscript [11]. Of the remaining 66 articles related to all other anti-inflammatory agents, 25 articles were excluded after detailed assessment, based on the inclusion/exclusion criteria. Thus, 41 articles 
were formally assessed for the present review. These 41 studies investigated the use of 15 anti-inflammatory agents (benzydamine, diphenhydramine, prostaglandin, misoprostol, immunoglobulin, corticosteroids, indomethacin, azelastine, mesalazine, disprin, orgotein, flurbiprofen, histamine, colchicine, and placentrex) for the prevention and/or treatment of oral mucositis. Summary data for these publications is presented in Table 1.

\section{Benzydamine mouthwash}

\section{Head and neck radiotherapy: prevention}

Six studies were reviewed [12-17]. The non-superiority of benzydamine mouthwash when compared with chlorhexidine was reported in one unblinded study, although formal recording of oral mucositis was not conducted [13]. Significantly lower pain ratings by patients who received benzydamine compared to placebo were reported in three randomized, double blind, placebo-controlled studies [12, 14, 15], resulting in fewer patients requiring systemic analgesics. Epstein and colleagues found that up to cumulative doses of $50 \mathrm{~Gy}$, subjects receiving benzydamine to prevent oral mucositis had significantly less erythema and ulceration and were more likely to remain ulcer-free as compared to subjects who received placebo mouthwash [15]. Based largely on the findings of this large well-designed multicenter study, benzydamine was recommended for the prevention of moderate-dose radiation-induced oral mucositis in the 2005 mucositis guidelines update [5]. A beneficial effect of benzydamine mouthwash was also reported in two subsequent studies, which further supported the previous recommendation $[16,17]$. In the study conducted by Cheng et al. (2006), oral mucositis was less severe, although the difference was not statistically significant, for the benzydamine group when compared with the chlorhexidine group. [16]. Patients in the benzydamine group required less supplemental analgesics. The total radiation dose varied between 54 and 68 Gy in both groups. In the study conducted by Kazemian et al. (2009), which included 81 patients and was randomized, double blind, and placebo controlled, benzydamine significantly reduced oral mucositis during radiotherapy. The frequency of oral mucositis $\geq$ grade 3 was significantly less in the benzydamine group when compared to the placebo. Benzydamine mouthwash was reported as effective, safe, and well tolerated. The mean total radiation dose was $61.69 \mathrm{~Gy}$ for the benzydamine and $64.02 \mathrm{~Gy}$ for the placebo group [17]. However, patients with grade 2 or higher mucositis were allowed to use other topical medications including diphenhydramine and nystatin. There was no adjustment for or comparison of the use of such concomitant medications that could potentially affect oral mucositis. These issues prevented the extension of the recommendation in favor of benzydamine to the population of head and neck cancer patients receiving radiation doses higher than $50 \mathrm{~Gy}$.

Previous guideline: The panel recommends benzydamine for prevention of radiation-induced mucositis in patients with head and neck cancer receiving moderate-dose radiation therapy.

New guideline: The panel recommends benzydamine mouthwash for the prevention of oral mucositis in patients with head and neck cancer receiving moderatedose radiation therapy (up to $50 \mathrm{~Gy}$ ), without concomitant chemotherapy.

\section{Head and neck radiotherapy: treatment}

Significant analgesic activity and alleviation of symptoms were observed in a double blind and placebo-controlled study, which tested benzydamine for the treatment of oral mucositis [18, 19]. The authors published results of the same patient cohort on two occasions, the preliminary findings in 1985, and the final results in 1986. Oral pain was the primary endpoint. The findings of this single study, despite the promising results, were not adequate to support establishment of a guideline for the use of benzydamine to treat oral mucositis during head and neck radiotherapy. Therefore, no guideline was possible.

\section{Head and neck chemoradiotherapy: prevention}

Three studies were reviewed [20-22]. Oral mucositis prevalence was lower and it appeared significantly later in patients treated with prophylactic benzydamine when compared with placebo in two studies [20,21]. In the first study investigating benzydamine by Prada and colleagues, methodology reporting was insufficient to determine whether chemotherapy was administered concurrently with radiotherapy [20]. In the second study by Prada and colleagues, in addition to chemoradiotherapy, patients also received chemotherapy or radiotherapy alone [21]. Pain and dysphagia were the primary endpoints in both those studies [20, 21]. The third study, conducted by Putwatana et al. (2009), was a prospective, randomized, single-blinded study, which compared benzydamine mouthwash with papayor drops [22]. Benzydamine was found inferior to papayor herb for preventing and relieving radiation-induced oral mucositis and pain. Fifty percent of patients in the benzydamine group and $56.67 \%$ of those in the papayor group received radiotherapy alone. Due to the insufficient data and the presence of concerns such as lack of double blinding, no guideline was possible regarding the use of benzydamine mouthwash for the prevention of oral mucositis in head and neck chemoradiotherapy. 


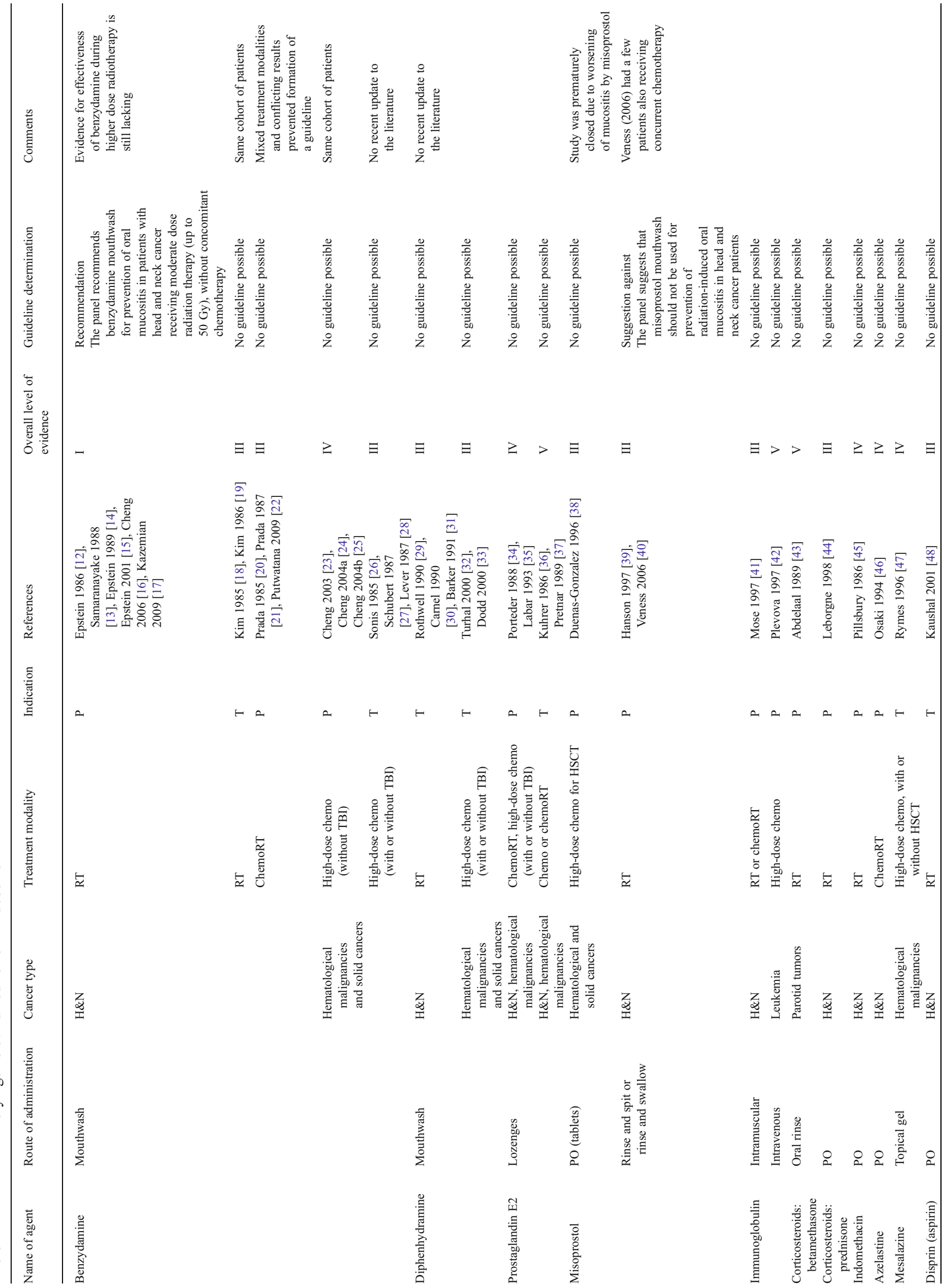


Three articles reporting results from the same cohort of pediatric patients, who received high-dose or combination chemotherapy for hematological malignancies or solid tumors, were reviewed [23-25]. The original study was a prospective, randomized, non-blinded, two-period crossover study that compared an oral care protocol containing benzydamine to chlorhexidine [23, 24]. In the 34 children evaluated, benzydamine was found inferior to chlorhexidine in the prevention of oral mucositis. The authors commented that the results need to be confirmed in a separate larger trial. The acceptance and tolerance of benzydamine was reported in the third article published in the series [25]. Benzydamine was found acceptable and well tolerated by children over the age of 6 years [25]. The insufficient data did not allow for any guideline for or against the use of benzydamine for the prevention of oral mucositis in patients receiving high-dose chemotherapy.

\section{High-dose chemotherapy: treatment}

Three studies were reviewed, two tested benzydamine in adults $[26,27]$ and one in children [28]. Patients had hematological cancers or solid tumors other than head and neck cancer, and received high-dose chemotherapy. Total body irradiation was included in the conditioning regimen in 17/44 patients in the study conducted by Schubert and colleagues [27]. Benzydamine was consistently more effective than placebo to relieve oral mucositis-associated pain, but the difference was not significant in this multicenter, double-blind, placebocontrolled trial [27]. In a small pilot study of nine patients by Sonis et al. (1985), palliation of pain was reported by seven study subjects [26]. Mucosal irritation and burning, possibly associated with the alcohol base of the mouthwash, was the most frequently reported toxicity. No patient dropped out, however, due to this toxicity $[26,27]$. In the pediatric study, three children were unwilling to tolerate the oral pain and irritation associated with benzydamine mouthwash contact and dropped out. Another three of four children who completed the protocol opted to continue therapy with the alternative mouthwash for pain, which included nystatin and lidocaine in sodium chloride [28]. Due to insufficient evidence and inconsistent results, the panel could not support any guideline for or against the use of benzydamine for the treatment of oral mucositis in patients receiving high-dose chemotherapy.

Diphenhydramine mouthwash

\section{Head and neck radiotherapy: treatment}

Three studies were reviewed [29-31]. Diphenhydramine, combined with hydrocortisone, nystatin, and tetracycline, 
reduced oral mucositis in a pilot, double-blind, placebocontrolled study of 12 patients conducted by Rothwell and colleagues [29]. Carnel et al. (1990) compared diphenhydramine, combined with kaolin-pectin, with different oral rinses in a pilot, double-blind study, which included 18 subjects, who received radiotherapy or chemoradiotherapy (number of patients in each treatment modality was not specified) [30]. No conclusions were drawn regarding the effectiveness of diphenhydramine [30]. Finally, no significant difference between groups was found in a prospective, double-blind, non-controlled study by Barker et al. (1991), which tested diphenhydramine, combined with kaolin-pectin, against sucralfate suspension in 12 patients [31]. Pain was the primary endpoint investigated [31]. Insufficient evidence, the concomitant use of confounding agents, and inconsistent results between studies did not allow for any guideline for or against the use of diphenhydramine for the treatment of oral mucositis in patients receiving head and neck radiotherapy.

\section{High-dose chemotherapy: treatment}

Two studies were reviewed [32, 33]. Turhal and colleagues conducted a non-controlled study, and found that diphenhydramine, combined with lidocaine and sodium bicarbonate, provided effective symptomatic relief [32]. Nine of the 31 patients evaluated had hematological malignancies and underwent HSCT. Four patients received radiotherapy, not otherwise specified. In the second article reviewed, Dodd et al. (2000) conducted a randomized, double-blinded study, where diphenhydramine combined with lidocaine and aluminum hydroxide (Maalox) was compared with two other mouthwashes, one of which was chlorhexidine $0.12 \%$ and the second was salt and soda in water [33]. The patients received chemotherapy for hematological malignancies and solid tumors. No significant differences in time for the cessation of the signs and symptoms of mucositis were observed among the three groups. Pain relief was the primary endpoint in both studies. Insufficient evidence, the concomitant use of confounding agents, and inconsistent results between the studies did not allow for any guideline for or against the use of diphenhydramine for the treatment of oral mucositis in patients receiving high-dose chemotherapy.

\section{Prostaglandin E2 lozenges}

\section{Head and neck chemoradiotherapy, high-dose chemotherapy: prevention}

Two papers were reviewed [34, 35]. Firstly, in 10 patients with oral cancers, who received chemoradiotherapy, prostaglandin E2 (PGE2) lozenges reduced pain and oral mucosal inflammation when compared with controls. No systemic absorption was found [34]. This study was blinded and placebo controlled, but subjects were not randomly assigned to PGE2 or placebo. Statistical analysis of results was not described. In the randomized, double-blind, placebocontrolled study by Labar and colleagues, PGE2 lozenges did not prevent oral mucositis in patients with leukemia, who received high-dose chemotherapy with or without TBI for HSCT [35]. A significantly higher incidence of HSV infections was also found in the prostaglandin group [35]. Due to insufficient evidence, the panel could not support any guideline for or against the use of topical PGE2 to prevent oral mucositis in these cancer settings.

\section{Head and neck chemotherapy or chemoradiotherapy, high-dose chemotherapy: treatment}

Two studies were reviewed [36, 37]. In a small, uncontrolled pilot study, topical PGE2 reduced pain and oral inflammation in 4/6 patients with oral mucositis [36]. PGE2 was also reported to prevent oral lesions in two more patients in the same report [36]. Five of the study patients had oral or oropharyngeal cancers. In the second study reviewed, prostaglandin lozenges promoted healing of oral mucositis in 9/11 patients when compared with three control subjects, who used antiseptic lozenges and gargles [37]. All patients had received chemotherapy with or without skull irradiation or high-dose chemotherapy prior to HSCT for the treatment of hematological malignancies. Randomization and blinding were not used, and there was no statistical analysis of the results. As such, insufficient evidence prevented the panel from being able to develop any guideline for or against the use of PGE2 for the treatment of oral mucositis in the above cancer settings.

\section{Misoprostol}

\section{High-dose chemotherapy: prevention}

In a study by Duenas-Gonzalez et al. (1996), the incidence and severity of oral mucositis was increased in patients with hematological and solid malignancies, who received highdose chemotherapy prior to HSCT and were treated with misoprostol tablets (nine patients) when compared with the placebo control group (seven patients) [38]. The study was prematurely closed because of the significant difference in the incidence and severity of oral mucositis favoring the placebo patients. No guideline was possible in this population due to the single study reviewed.

\section{Head and neck radiotherapy: prevention}

Misoprostol oral rinse was studied in a randomized doubleblind placebo controlled study in head and neck cancer 
patients, receiving post-operative radiotherapy at one of two study sites. Data from one study site showed a decrease in the mean mucositis scores in the misoprostol group at the fourth and fifth week of RT, with no significant decrease seen at the sixth and seventh weeks. No benefit of misoprostol was seen at the second site or when data from both sites were analyzed together [39]. In another study of 83 subjects, misoprostol, used as an oral rinse before swallowing, did not reduce the incidence of oral mucositis in head and neck cancer patients, who received radiotherapy or chemoradiotherapy [40]. There was also no difference in oropharyngeal/oral soreness and general well-being, as reported by study subjects. Both studies were randomized, double blind, and placebo-controlled. The overall negative results from both studies supported a new suggestion against the use of misoprostol for the prevention of radiationinduced oral mucositis in head and neck cancer patients.

\section{Previous guideline None}

New guideline The panel suggests that misoprostol mouthwash should not be used for the prevention of radiationinduced oral mucositis in head and neck cancer patients.

Immunoglobulin

\section{Head and neck radiotherapy or chemoradiotherapy/ intramuscular administration: prevention}

Weekly intramuscular administration of immunoglobulin in head and neck cancer patients, who received radiotherapy or chemoradiotherapy reduced, though not significantly, oral mucositis severity when compared with controls [41]. The study was not randomized nor double blinded. No guideline was possible due to insufficient evidence.

\section{High-dose chemotherapy/i.v. administration: prevention}

A single, intravenous administration of immunoglobulin reduced oral mucositis in two children with leukemia, who received high-dose chemotherapy [42]. Those two children had developed grade 3 oral mucositis following previous chemotherapy without immunoglobulin. No guideline was possible due to insufficient evidence.

Corticosteroids

\section{Head and neck radiotherapy: prevention}

6a Betamethasone rinse, without swallowing, prevented oral mucositis in a total of five study patients (100\%) with parotid tumors, who were irradiated [43].
Methodology was not adequately described and controls were not used.

$6 \mathrm{~b}$ Prednisone tablets given orally, $40 \mathrm{mg} /$ day, administered for 8 days following the initiation of radiotherapy in 32 head and neck cancer patients, did not reduce the intensity or duration of oral mucositis in a randomized, double-blind, placebo-controlled study [44]. These two studies provided insufficient evidence to support any guideline for or against the use of corticosteroids for prevention of oral mucositis due to cancer therapy.

Indomethacin PO (tablets given orally)

\section{Head and neck radiotherapy: prevention}

Daily per os indomethacin significantly delayed the onset of grade 3 oral mucositis in 10 head and neck cancer patients, who received radiotherapy when compared to eight controls [45]. The study was prospective, randomized, double blind, and placebo controlled. The grades of oral mucositis were not adequately described and the number of study patients was small. As such, there was insufficient evidence to support any guideline for the use of indomethacin for the prevention of oral mucositis due to cancer therapy.

Azelastine PO (tablets given orally)

\section{Head and neck chemoradiotherapy: prevention}

Azelastine hydrochloride is thought to have an antiinflammatory action, based on its leukocyte suppression effect. Daily per os azelastine significantly reduced the severity of oral mucositis in 37 patients with oral cancer, treated with chemoradiotherapy, when compared with 26 controls [46]. The study was prospective, randomized, and controlled but not blinded. The beneficial effect of azelastine was attributed to the anti-oxidant action of the agent. The authors suggested further studies. This single study provided insufficient evidence to support any guideline for the use of azelastine for the prevention of oral mucositis due to cancer therapy.

Mesalazine topical gel

\section{High-dose chemotherapy: treatment}

Some symptomatic relief following daily topical mesalazine (5-aminosalicylic acid) gel was reported, using a questionnaire, by 10 of 12 patients with hematological malignancies, treated with high-dose chemotherapy with or without HSCT [47]. The study was open-label, non-randomized, and oral 
mucositis was not formally graded. This study provided insufficient evidence to support any guideline for the use of mesalazine for the treatment of oral mucositis due to cancer therapy.

Disprin PO (tablets dissolved in water)

\section{Head and neck radiotherapy: treatment}

Guideline No guideline possible. Disprin (aspirin) tablets, dissolved in water and combined with betamethasone drops, were tested in the treatment of radiation-induced oral mucositis. The agent did not show a beneficial effect on oral mucositis when compared with placentrex extracts [48]. The study was prospective and randomized. The blinded method was not used, statistical analysis was not shown, and oral mucositis was not adequately recorded. This single study provided insufficient evidence to support any guideline for the use of Disprin/aspirin for the treatment of oral mucositis due to cancer therapy.

Orgotein intramuscular injection

\section{Head and neck radiotherapy or chemoradiotherapy: treatment}

Orgotein (superoxide dismutase) catalyzes the dismutation of damaging superoxide radicals, acting as an antioxidant. In a retrospective study, orgotein administered three times per week appeared to reduce the severity of oral mucositis in 40 head and neck cancer patients treated with radiotherapy or chemoradiotherapy [49]. A $92.5 \%$ overall response was reported. No controls were used. The authors suggested additional, controlled studies to define the exact role of orgotein in the treatment of oral mucositis. To date, there is insufficient evidence from this single study to support any guideline for the use of orgotein for the treatment of oral mucositis due to cancer therapy.

\section{Flurbiprofen tooth patch}

\section{Head and neck radiotherapy: prevention}

Flurbiprofen, administered as a tooth patch that released drug locally in the oral cavity, did not reduce the severity and duration of oral mucositis in 12 head and neck cancer patients treated with RT, when compared with historical controls [50]. However, the onset of pseudomembranous/ ulcerative mucositis occurred significantly later in the flurbiprofen group. This single study provided insufficient evidence to support any guideline regarding the use of flurbiprofen for the prevention of oral mucositis due to cancer therapy.

Histamine topical gel

High-dose chemotherapy: prevention

Histamine dihydrochloride did not prevent oral mucositis in patients with hematological and other cancers, who received chemotherapy and HSCT, with or without total body irradiation (TBI) [51]. Histamine gel was safe without severe adverse events. A total of 45 patients were enrolled in the study which was prospective, longitudinal, placebo controlled, and double blind. Further studies were suggested to compare various dosages of the agent in HSCT patients in order to establish an optimal clinical effect with minimum side effects. Insufficient evidence provided by this single study did not allow for the generation of any guideline regarding the use of histamine for the prevention of oral mucositis due to cancer therapy.

Colchicine mouthwash

\section{High-dose chemotherapy: treatment}

Colchicine is an anti-gout agent that has anti-inflammatory activity. Colchicine significantly reduced the severity and duration of oral mucositis in 40 patients with hematological malignancies treated with high-dose chemotherapy, when compared with 42 historical controls who used saline mouthwash [52]. Colchicine was well tolerated. This single study provided insufficient evidence to support any guideline for the use of colchicine for the treatment of oral mucositis due to cancer therapy.

Placentrex intramuscular injection

\section{Head and neck radiotherapy: treatment}

Placentrex (human placental extract) has been suggested to have anti-inflammatory, analgesic, and immunostimulant properties. It was administered intramuscularly to 60 head and neck cancer patients with radiotherapy-induced oral mucositis, and resulted in decreased oral pain when compared with 60 controls, who had received disprin gargles and betamethasone [48]. The study was prospective and randomized. However, the blinded method was not used, statistical analysis was not shown, and oral mucositis was not adequately recorded. Insufficient evidence was provided by this single study to support any guideline for the use of placentrex for the treatment of oral mucositis due to cancer therapy. 


\section{Discussion}

Several studies have demonstrated that the administration of chemotherapy or radiation therapy results in a pronounced inflammatory response in the oral mucosa. For example, in a hamster cheek pouch model of radiation mucositis, it has been demonstrated that peak clinical mucosal injury is accompanied by a significant increase in inflammatory cell infiltrate as compared to baseline. Further, mRNA levels of TNF- $\alpha$ and IL- $1 \beta$ in the oral mucosal tissue correlated with severity of mucosal injury [53]. In a human study, levels of cyclooxygenase-2 (COX-2) and nuclear factor kappa-B $(\mathrm{NFkB})$ in the oral mucosa were significantly increased following cytotoxic chemotherapy [54]. These and other studies have provided a rationale for testing antiinflammatory agents for the prevention or treatment of oral mucositis.

Benzydamine hydrochloride is a non-steroidal antiinflammatory drug (NSAID), which has been shown to inhibit inflammatory cytokine production, such as TNF- $\alpha$ and IL-1 $\beta[55,56]$. In vivo treatment with benzydamine protected mice against lipopolysaccharide (LPS)-induced lethality. Protection against LPS toxicity was associated with a marked reduction of serum levels of TNF- $\alpha$ and IL$1 \beta$. The previous version of our guidelines included a recommendation for the use of benzydamine mouthwash to prevent oral mucositis in head and neck cancer patients, who receive moderate-dose radiotherapy [4-6, 12, 14-17]. This was based on evidence from studies showing a benefit in radiation doses up to $50 \mathrm{~Gy}$, in patients who did not receive concomitant chemotherapy. For this update, we reviewed two additional studies in this patient population. However, these studies did not allow the extension of this recommendation to patients receiving over 50 Gy radiation. Similarly, in other cancer treatment settings, including head and neck cancer patients receiving concomitant chemoradiation, the evidence did not support the development of any guidelines. Some studies reported intolerance to the topical benzydamine, with the alcohol base considered a major cause of noncompliance in children [28]. Welldesigned studies with new formulations of benzydamine would clarify the potential promising effect of this agent in various cancer populations.

In general, NSAIDS are known to have antiinflammatory properties, including suppression of the cyclooxygenase pathway and inhibition of production of proinflammatory prostaglandins. A number of NSAIDS have been evaluated for oral mucositis, including systemic indomethacin and aspirin. We also reviewed studies on the use of topical mesalazine and flurbiprofen. However, these single studies of different NSAIDS had inconsistent results and no clear conclusion could be drawn. Corticosteroids are more potent anti-inflammatory agents than NSAIDS. One uncontrolled case series of topical steroids suggested a beneficial effect in radiation-induced oral mucositis. But a randomized, double-blind, placebo-controlled study of systemic prednisone demonstrated no benefit in a similar population [44].

Misoprostol is a synthetic analog of prostaglandin E1 (PGE1), which has anti-inflammatory properties. Misoprostol also protects the mucosa and has been approved for reducing the risk of gastric ulcers induced by NSAID use. However, the studies we reviewed supported the development of a new suggestion against the use of misoprostol mouthwash to prevent radiation-induced oral mucositis in head and neck cancer patients [38-40]. We reviewed a single study of misoprostol in chemotherapy-induced mucositis, which was prematurely closed due to more severe mucositis in the misoprostol group. No guideline was possible in chemotherapy patients based on this one study. However, a recent study by Lalla et al., published after the cut-off date for this review, also indicates a lack of efficacy of this agent for chemotherapy-induced oral mucositis [57]. Unlike PGE1, prostaglandin E2 is considered to be proinflammatory, but is also cytoprotective and promotes healing of gastric ulcerations. Interestingly, three of four studies of topical PGE2 reported a positive effect. However, these were uncontrolled preliminary studies. The only randomized double-blind placebo-controlled study of topical PGE2 found no difference in the incidence or duration of severe oral mucositis and a higher incidence of HSV infection in the PGE2 group [35].

Diphenhydramine HCL is an antihistamine which also has topical and local anesthetic properties. It is a common component of various combination rinses that are called "Magic" or "Miracle" mouthwashes. Although a number of studies examined the use of diphenhydramine for mucositis, it was always used in combination with other agents that made it difficult to definitively ascribe any benefit or lack thereof to diphenhydramine. Further, the results of these studies were mixed. Mild pain relief can be expected with topical diphenhydramine use, but the evidence does not support a direct effect of this antihistaminic on the prevention or treatment of mucositis lesions. Related to this, the use of topical histamine was also found to have no effect on oral mucositis in patients receiving high-dose chemotherapy for HSCT [51].

Despite the lack of clear evidence supporting the use of any anti-inflammatory agent other than benzydamine, the use of anti-inflammatory agents continues to be a promising strategy for the prevention and treatment of oral mucositis. Preliminary studies of a few anti-inflammatory agents have suggested potential benefit. However, definitive conclusions were hampered by flawed study designs, non-homogeneous patient populations and treatment modalities, small numbers of study subjects, varied modes of administration of study 
drug, administration in combination with other potentially active agents, intolerance of the vehicle/base leading to noncompliance, and other factors. Additional welldesigned studies are needed to examine the use of antiinflammatory agents for oral mucositis in various cancer treatment settings.

Disclosures The Mucositis Guidelines Update was sponsored by Helsinn Healthcare S.A., Switzerland and BioAlliance Pharma, France. Per MASCC policy, no industry representatives had any role in the development of the guidelines. The authors have full control of all primary data and agree to allow the Journal to review these data if requested.

\section{References}

1. Sonis ST (1998) Mucositis as a biological process: a new hypothesis for the development of chemotherapy-induced stomatotoxicity. Oral Oncol 34:39-43

2. Sonis ST, Elting LS, Keefe D, Peterson DE, Schubert M, HauerJensen M, Bekele BN, Raber-Durlacher J, Donnelly JP, Rubenstein EB (2004) Perspectives on cancer therapy-induced mucosal injury: pathogenesis, measurement, epidemiology, and consequences for patients. Cancer 100(9 Suppl):1995-2025

3. Xanthinaki A, Nicolatou-Galitis O, Athanassiadou P, Gonidi M, Kouloulias V, Sotiropoulou-Lontou A, Pissakas G, Kyprianou K, Kouvaris J, Patsouris E (2008) Apoptotic and inflammation markers in oral mucositis in head and neck cancer patients receiving radiotherapy: preliminary report. Support Care Cancer 16(9):1025-1033

4. Rubenstein EB, Peterson DE, Schubert M, Keefe D, McGuire D, Epstein J, Elting LS, Fox PC, Cooksley C, Sonis ST (2004) Clinical practice guidelines for the prevention and treatment of cancer therapy-induced oral and gastrointestinal mucositis. Cancer 100(9 Suppl):2026-2046

5. Lalla RV, Schubert MM, Bensadoun RJ, Keefe D (2006) Antiinflammatory agents in the management of alimentary mucositis. Support Care Cancer 14(6):558-565

6. Keefe DM, Schubert MM, Elting LS, Sonis ST, Epstein JB, RaberDurlacher JE, Migliorati CA, McGuire DB, Hutchins RD, Peterson DE (2007) Updated clinical practice guidelines for the prevention and treatment of mucositis. Cancer 109(5):820-831

7. Bowen JM, Elad S, Hutchins RD, Lalla RV (2013) Methodology for the MASCC/ISOO Mucositis Clinical Practice Guidelines Update. Support Care Cancer 21:303-308

8. Elad S, Bowen J, Zadik Y, Lalla RV (2013) Development of the MASCC/ISOO Clinical Practice Guidelines for Mucositis: considerations underlying the process. Support Care Cancer 21:309-312

9. Hadorn DC, Baker D, Hodges JS, Hicks N (1996) Rating the quality of evidence for clinical practice guidelines. J Clin Epidemiol 49(7):749-754

10. Somerfield MR, McCrae RR (2000) Stress and coping research. Methodological challenges, theoretical advances, and clinical applications. Am Psychol 55(6):620-6259

11. Nicolatou-Galitis O, Sarri T, Bowen J, Di Palma M, Kouloulias V, Niscola P et al (2013) Systematic review of amifostine for the management of oral mucositis in cancer patients. Support Care Cancer 21:357-364
12. Epstein JB, Stevenson-Moore P (1986) Benzydamine hydrochloride in prevention and management of pain in oral mucositis associated with radiation therapy. Oral Surg Oral Med Oral Pathol 62:145-148

13. Samaranayake LP, Robertson AG, MacFarlane TW, Hunter IP, MacFarlane G, Soutar DS, Ferguson MM (1988) The effect of chlorhexidine and benzydamine mouthwashes on mucositis induced by therapeutic irradiation. Clin Radiol 39:291-294

14. Epstein JB, Stevenson-Moore P, Jackson S, Mohamed JH, Spinelli JJ (1989) Prevention of oral mucositis in radiation therapy: a controlled study with benzydamine hydrochloride rinse. Int $\mathrm{J}$ Radiat Oncol Biol Phys 16:1571-1575

15. Epstein JB, Silverman S Jr, Paggiarino DA, Crockett S, Schubert MM, Senzer NN, Lockhart PB, Gallagher MJ, Peterson DE, Leveque FG (2001) Benzydamine $\mathrm{HCl}$ for prophylaxis of radiation-induced oral mucositis: results from a multicenter, randomized, double-blind, placebo-controlled clinical trial. Cancer 92:875-885

16. Cheng KF, Ka Tsui Yuen J (2006) A pilot study of chlorhexidine and benzydamine oral rinses for the prevention and treatment of irradiation mucositis in patients with head and neck cancer. Cancer Nurs 29:423-430

17. Kazemian A, Kamian S, Aghili M, Hashemi FA, Haddad P (2009) Benzydamine for prophylaxis of radiation-induced oral mucositis in head and neck cancers: a double-blind placebo-controlled randomized clinical trial. Eur J Cancer Care 18:174-178

18. Kim JH, Chu F, Lakshmi V, Houde R (1985) A clinical study of benzydamine for the treatment of radiotherapy-induced mucositis of the oropharynx. Int J Tissue React 7:215-218

19. Kim JH, Chu FC, Lakshmi V, Houde R (1986) Benzydamine HCl, a new agent for the treatment of radiation mucositis of the oropharynx. Am J Clin Oncol 9:132-134

20. Prada A, Lozza L, Moglia D, Sala L, Chiesa F (1985) Effects of benzydamine on radio-polychemotherapeutic mucositis of the oral cavity. Int J Tissue React 7:237-239

21. Prada A, Chiesa F (1987) Effects of benzydamine on the oral mucositis during antineoplastic radiotherapy and/or intra-arterial chemotherapy. Int J Tissue React 9:115-119

22. Putwatana $P$, Sanmanowong $P$, Oonprasertpong L, Junda $T$, Pitiporn S, Narkwong L (2009) Relief of radiation-induced oral mucositis in head and neck cancer. Cancer Nurs 32:82-87

23. Cheng KK, Chang AM (2003) Palliation of oral mucositis symptoms in pediatric patients treated with cancer chemotherapy. Cancer Nurs 26:476-484

24. Cheng KK, Chang AM, Yuen MP (2004) Prevention of oral mucositis in paediatric patients treated with chemotherapy; a randomised crossover trial comparing two protocols of oral care. Eur J Cancer 40:1208-1216

25. Cheng KK (2004) Children's acceptance and tolerance of chlorhexidine and benzydamine oral rinses in the treatment of chemotherapy-induced oropharyngeal mucositis. Eur J Oncol Nurs 8:341-349

26. Sonis ST, Clairmont F, Lockhart PB, Connolly SF (1985) Benzydamine HCL in the management of chemotherapy-induced mucositis I. Pilot study. J Oral Med 40:67-71

27. Schubert MM, Newton RE (1987) The use of benzydamine $\mathrm{HCl}$ for the management of cancer therapy-induced mucositis: preliminary report of a multicentre study. Int J Tissue React 9:99-103

28. Lever SA, Dupuis LL, Chan HS (1987) Comparative evaluation of benzydamine oral rinse in children with antineoplastic-induced stomatitis. Drug Intell Clin Pharm 21:359-361

29. Rothwell BR, Spektor WS (1990) Palliation of radiation-related mucositis. Spec Care Dentist 10:21-25

30. Carnel SB, Blakeslee DB, Oswald SG, Barnes M (1990) Treatment of radiation- and chemotherapy-induced stomatitis. Otolaryngol Head Neck Surg 102:326-330 
31. Barker G, Loftus L, Cuddy P, Barker B (1991) The effects of sucralfate suspension and diphenhydramine syrup plus kaolinpectin on radiotherapy-induced mucositis. Oral Surg Oral Med Oral Pathol 71:288-293

32. Turhal NS, Erdal S, Karacay S (2000) Efficacy of treatment to relieve mucositis-induced discomfort. Support Care Cancer 8:55-58

33. Dodd MJ, Dibble SL, Miaskowski C, MacPhail L, Greenspan D, Paul SM, Shiba G, Larson P (2000) Randomized clinical trial of the effectiveness of 3 commonly used mouthwashes to treat chemotherapy-induced mucositis. Oral Surg Oral Med Oral Pathol Oral Radiol Endod 90:39-47

34. Porteder H, Rausch E, Kment G, Watzek G, Matejka M, Sinzinger $\mathrm{H}$ (1988) Local prostaglandin E2 in patients with oral malignancies undergoing chemo- and radiotherapy. J Craniomaxillofac Surg $16: 371-374$

35. Labar B, Mrsic M, Pavletic Z, Bogdanic V, Nemet D, Aurer I, Radman I, Filipovic-Grcic N, Sertic D, Kalenic S et al (1993) Prostaglandin E2 for prophylaxis of oral mucositis following BMT. Bone Marrow Transplant 11:379-382

36. Kuhrer I, Kuzmits R, Linkesch W, Ludwig H (1986) Topical PGE2 enhances healing of chemotherapy-associated mucosal lesions. Lancet 1(8481):623

37. Pretnar J, Glazar D, Mlakar U, Modic M (1989) Prostaglandin E2 in the treatment of oral mucositis due to radiochemotherapy in patients with haematological malignancies. Bone Marrow Transplant 4(Suppl 3): 106

38. Duenas-Gonzalez A, Sobrevilla-Calvo P, Frias-Mendivil M, Gallardo-Rincon D, Lara-Medina F, Aguilar-Ponce L, MirandaLopez E, Zinser-Sierra J, Reynoso-Gomez E (1996) Misoprostol prophylaxis for high-dose chemotherapy-induced mucositis: a randomized double-blind study. Bone Marrow Transplant 17:809-812

39. Hanson WR, Marks JE, Reddy SP, Simon S, Mihalo WE, Tova Y (1997) Protection from radiation-induced oral mucositis by a mouth rinse containing the prostaglandin $\mathrm{E} 1$ analog, misoprostol: a placebo controlled double blind clinical trial. Adv Exp Med Biol 400B:811-818

40. Veness MJ, Foroudi F, Gebski V, Timms I, Sathiyaseelan Y, Cakir B, Tiver KW (2006) Use of topical misoprostol to reduce radiation-induced mucositis: results of a randomized, doubleblind, placebo-controlled trial. Australas Radiol 50:468-474

41. Mose S, Adamietz IA, Saran F, Thilmann C, Heyd R, Knecht R, Bottcher HD (1997) Can prophylactic application of immunoglobulin decrease radiotherapy-induced oral mucositis? Am J Clin Oncol 20:407-411

42. Plevova P, Blazek B (1997) Intravenous immunoglobulin as prophylaxis of chemotherapy-induced oral mucositis. J Natl Cancer Inst 89:326-327

43. Abdelaal AS, Barker DS, Fergusson MM (1989) Treatment for irradiation-induced mucositis. Lancet 1(8629):97

44. Leborgne JH, Leborgne F, Zubizarreta E, Ortega B, Mezzera J (1998) Corticosteroids and radiation mucositis in head and neck cancer. A double-blind placebo-controlled randomized trial. Radiother Oncol 47:145-148
45. Pillsbury HC 3rd, Webster WP, Rosenman J (1986) Prostaglandin inhibitor and radiotherapy in advanced head and neck cancers. Arch Otolaryngol Head Neck Surg 112:552-553

46. Osaki T, Ueta E, Yoneda K, Hirota J, Yamamoto T (1994) Prophylaxis of oral mucositis associated with chemoradiotherapy for oral carcinoma by Azelastine hydrochloride (Azelastine) with other antioxidants. Head Neck 16:331-339

47. Rymes N, Glick L, Holmes JA (1996) Topical mesalazine in the treatment of chemotherapy and radiotherapy-induced oral mucositis. Bone Marrow Transplant 18:484

48. Kaushal V, Verma K, Manocha S, Hooda HS, Das BP (2001) Clinical evaluation of human placental extract (placentrex) in radiation-induced oral mucositis. Int J Tissue React 23:105-110

49. Valencia J, Velilla C, Urpegui A, Alvarez I, Llorens MA, Coronel P, Polo S, Bascon N, Esco R (2002) The efficacy of orgotein in the treatment of acute toxicity due to radiotherapy on head and neck tumors. Tumori 88:385-389

50. Stokman MA, Spijkervet FK, Burlage FR, Roodenburg JL (2005) Clinical effects of flurbiprofen tooth patch on radiation-induced oral mucositis. A pilot study. Support Care Cancer 13:42-48

51. Elad S, Ackerstein A, Bitan M, Shapira MY, Resnick I, Gesundheit B, Cohen Y, Diss O, Barak D, Dray L, Or R (2006) A prospective, double-blind phase II study evaluating the safety and efficacy of a topical histamine gel for the prophylaxis of oral mucositis in patients post hematopoietic stem cell transplantation. Bone Marrow Transplant 37:757-762

52. Garavito AA, Cardona AF, Reveiz L, Ospina E, Yepes A, Ospina V (2008) Colchicine mouth washings to improve oral mucositis in patients with hematological malignancies: a clinical trial. Palliat Support Care 6:371-376

53. Sonis ST, Peterson RL, Edwards LJ, Lucey CA, Wang L, Mason L et al (2000) Defining mechanisms of action of interleukin-11 on the progression of radiation-induced oral mucositis in hamsters. Oral Oncol 36:373-381

54. Logan RM, Gibson RJ, Sonis ST, Keefe DMK (2007) Nuclear factor- $\mathrm{kB}(\mathrm{NF}-\mathrm{kB})$ and cyclooxygenase-2 (COX-2) expression in the oral mucosa following cancer chemotherapy. Oral Oncol 43:395-401

55. Sironi M, Pozzi P, Polentarutti N, Benigni F, Coletta I, Guglielmotti A, Milanese C, Ghezzi P, Vecchi A, Pinza M, Mantovani A (1996) Inhibition of inflammatory cytokine production and protection against endotoxin toxicity by benzydamine. Cytokine 8(9):710-716

56. Sironi M, Milanese C, Vecchi A, Polenzani L, Guglielmotti A, Coletta I, Landolfi C, Soldo L, Mantovani A, Pinza M (1997) Benzydamine inhibits the release of tumor necrosis factor-alpha and monocyte chemotactic protein-1 by Candida albicans-stimulated human peripheral blood cells. Int J Clin Lab Res 27:118-122

57. Lalla RV, Gordon GB, Schubert M, Silverman S Jr, Hutten M, Sonis ST et al (2012) A randomized, double-blind, placebocontrolled trial of misoprostol for oral mucositis secondary to high-dose chemotherapy. Support Care Cancer 20:1797-1804 